\title{
ON MEROMORPHIC FUNCTIONS OF BOUNDED CHARACTERISTIC
}

DAVID A. STORVICK ${ }^{1}$

1. Introduction. Let $w=f(z)$ be meromorphic in $|z|<1$, and let the characteristic function $T(r, f)$ be bounded, i.e., $T(r, f)=O(1)$ in $|z|<1$. If the radial limit values $f^{*}\left(e^{i \theta}\right)=\lim _{r \rightarrow 1} f\left(r e^{i \theta}\right)$ (which exist and are finite for almost all $e^{i \theta}$ on $|z|=1$ by the Fatou-Nevanlinna theorem $)^{2}$ have modulus one for almost all $e^{i \theta}$, we shall say that $f(z)$ is of class (B) in $|z|<1$; if, in addition, a function $f(z)$ of class (B) is bounded, $|f(z)|<1$ in $|z|<1$, we shall say that $f(z)$ is of class (A) in $|z|<1$. The principal result concerning functions of class (A) is the following theorem of W. Seidel [12, p. 205] which we state for reference in the sequel.

THEOREM 1. Let $w=f(z)$ be of class (A) in $|z|<1$, and take there the value $\alpha,|\alpha|<1$ at most a finite number of times. Then, unless $f(z)$ reduces identically to a finite Blaschke product in $|z|<1$ giving the most general $(m, 1)$ conformal map of $|z|<1$ onto $|w|<1$, there exists at least one radius $\theta=\theta_{0}$, such that $f^{*}\left(e^{i \theta_{0}}\right)=\alpha$.

It has been shown by G. Hössjer $[5$, p. 55] that the radial limit values of a nonconstant function $f(z)$ of class (A) comprise a set $E$ of measure $2 \pi$ on $|w|=1$. It follows almost trivially from Theorem 1 that every point of $|w|=1$ belongs to the set $E$. Indeed, if $e^{i \lambda}$ is an arbitrary point of $|w|=1$, the function

$$
g(z)=\exp \frac{f(z)+e^{i \lambda}}{f(z)-e^{i \lambda}}
$$

is of class (A) in $|z|<1$ and omits the value 0 . By Theorem 1 , there exists at least one radius $\theta=\theta_{0}$, for which $g^{*}\left(e^{i \theta_{0}}\right)=\lim _{r \rightarrow 1} g\left(r e^{i \theta_{0}}\right)=0$, from which it follows that $f^{*}\left(e^{i \theta_{0}}\right)=e^{i \lambda}$.

In $\$ 2$ we shall prove the analogue of this extension of Hössjer's theorem for functions of class (B). In $\$ 3$ we shall discuss another class of meromorphic functions which has been studied recently by $\mathrm{O}$.

Presented to the Society, June 19, 1954 and November 27, 1954; received by the editors January 24, 1955, and, in revised form, February 20, 1956.

1 This paper forms Part I of the author's doctoral dissertation written at the University of Michigan under the direction of Professor A. J. Lohwater. ff.].

${ }^{2}$ For this and the general theory of such meromorphic functions, see [11, pp. 208 
Lehto $[6 ; 7 ; 8]$ and M. Tsuji [14]: Let $f(z)$ be meromorphic in $|z|<1$, and let the values which $f(z)$ assumes in $|z|<1$ lie in a domain $G$ whose boundary $\Gamma$ has positive logarithmic capacity, so that by $[11$, p. 213] $f(z)$ is of bounded characteristic in $|z|<1$. Furthermore, we shall assume that $f^{*}\left(e^{i \theta}\right)$ belongs to $\Gamma$ for almost all $e^{i \theta}$ on $|z|=1$. In what follows, we shall say that a function $f(z)$ with these properties is of class $(L)$ in $|z|<1$. Lehto has not only extended the result of Seidel (Theorem 1) by showing that any value $a$ lying in $G$ which is omitted by $f(z)$ is a radial limit value of $f(z)$, but also that any $a$ in $G$ for which the "deficiency"

$$
\Phi(a)=\frac{1}{2 \pi} \lim _{r \rightarrow 1} \int_{0}^{2 \pi} g\left(f\left(r e^{i \theta}\right), a\right) d \theta
$$

is positive, where $g(w, a)$ is the Green's function of $G$ with singularity at $w=a$, is also a radial limit value of $f(z)$.

In $\S 3$ we shall extend the result of Hössjer to functions of class $(L)$ by showing that every point of $\Gamma$ which is arcwise accessible from $G$ is also a radial limit value of $f(z)$, and that, except for two special cases, every accessible point of $\Gamma$ is a radial limit of $f(z)$ infinitely often.

2. We shall assume in this section that $f(z)$ is a nontrivial function of class (B), i.e., that neither $f(z)$ nor $1 / f(z)$ reduces to a function of class (A). We shall say that a number $\alpha$ is in the range of $f(z)$ at a point $P$ of $|z|=1$ if $\alpha$ is assumed by $f(z)$ in every neighborhood of $P$. The number $\alpha$ will be said to be in the range of $f(z)$ in $|z|<1$ if it is assumed infinitely often in $|z|<1$.

THEOREM 2. If $w=f(z)$ is a nontrivial function of class (B), then either every point of $|w|=1$ which is not in the range of $f(z)$ is an asymptotic value of $f(z)$, or else $f(z)$ is a p-valent function mapping $|z|<1$ onto a simply-connected region consisting of the w-plane slit along an arc of $|w|=1$.

We assume first that the point $e^{i \lambda}$ is not in the range of $f(z)$ in $|z|<1$, i.e., $e^{i \lambda}$ is assumed at most finitely often by $f(z)$ in $|z|<1$. Then the function

$$
\phi(z)=\exp \frac{f(z)+e^{i \lambda}}{f(z)-e^{i \lambda}}
$$

is analytic in some annulus $\rho<|z|<1$, and omits the value 0 . Since, by the Riesz-Nevanlinna Theorem [11, p. 209], $\lim _{\inf _{r \rightarrow 1}}\left|f(z)-e^{i \lambda}\right|$ can be zero on at most a set of measure zero of $|z|=1$, the function 
$\phi(z)$ has the property that its radial limits exist and have modulus 1 almost everywhere on $|z|=1$.

Now if $e^{i \lambda}$ is not assumed at all in $|z|<1$ by $f(z)$, the function $\phi(z)$ in (2) is analytic in $|z|<1$ and omits there the value 0 . Since $\lim _{r \rightarrow 1}\left|\phi\left(r e^{i \theta}\right)\right|=1$ almost everywhere on $|z|=1$, it follows from a theorem of Lohwater $[9$, p. 248] that either 0 or $\infty$ is an asymptotic value of $\phi(z)$, so that $e^{i \lambda}$ is an asymptotic value of $f(z)$.

Suppose next that $e^{i \lambda}$ is actually assumed by $f(z)$ in $|z|<1$, but by hypothesis, only finitely often. From a recent extension of Schwarz's reflection principle by Lohwater [10] we have that a necessary and sufficient condition that $\phi(z)$ can be continued analytically beyond $|z|=1$ is that $\phi(z)$ admits neither 0 nor $\infty$ as an asymptotic value. If $f(z)$ is not analytic at every point of $|z|=1$, there exists an $\operatorname{arc} L$, lying in $|z|<1$ and terminating at a point $e^{i \theta_{0}}$ such that, as $z \rightarrow e^{i \theta_{0}}$ along $L, \phi(z)$ tends to 0 or $\infty$, whence $f(z) \rightarrow e^{i \lambda}$ along $L$, so that $e^{i \lambda}$ is an asymptotic value of $f(z)$.

On the other hand, if $\phi(z)$ is analytic on $|z|=1$, then $f(z)$ can have only a finite number of zeros and poles in $|z|<1$. If for example, $f(z)$ has an infinite number of zeros in $|z|<1$, let $\left\{z_{k}\right\}, k=1,2, \cdots$, denote a subsequence of these zeros converging to some point $e^{i \theta_{0}}$ on $|z|=1$. On this subsequence we must have $\lim _{k \rightarrow \infty} \phi\left(z_{k}\right)=\phi\left(e^{i \theta_{0}}\right)$ $=e^{-1}$ which is a contradiction since $\phi(z)$ must be analytic with modulus 1 everywhere on $|z|=1$. We consider, finally, the PoissonStieltjes representation $[11$, p. 201] of $w=f(z)$.

(3) $f(z)=\prod_{j=1}^{m} \frac{\alpha_{j}-z}{1-\bar{\alpha}_{j} z} \prod_{k=1}^{n} \frac{1-\bar{\beta}_{k} z}{\beta_{k}-z} \exp \left[\frac{1}{2 \pi} \int_{0}^{2 \pi} \frac{e^{i t}+z}{e^{i t}-z} d \mu(t)+i \gamma\right]$,

where $\alpha_{j}$ and $\beta_{k}$ are the zeros and poles, respectively of $f(z), \gamma$ is a real constant, and $\mu(t)$ is of bounded variation on $0 \leqq t \leqq 2 \pi$.

Since the finite products in (3) have modulus 1 everywhere on $|z|=1$, it follows from an argument identical to that used in [9, p. 246] that $\mu(t)$ is identically constant, so that $f(z)$ reduces to a quotient of two finite Blaschke products,

$$
f(z)=e^{i \gamma} \prod_{j=1}^{m} \frac{\alpha_{j}-z}{1-\bar{\alpha}_{j} z} \prod_{k=1}^{n} \frac{1-\bar{\beta}_{k} z}{\beta_{k}-z}, \quad n, m \geqq 1 .
$$

It cannot happen that the domain $G$ in the $w$-plane onto which $f(z)$ maps $|z|<1$, is multiply connected, for otherwise $f(z)$ must then assume infinitely often in $|z|<1$ every value of $G$; in particular, $e^{i \lambda}$ would then be in the range of $f(z)$, a contradiction. It is easy to see that the simply-connected region $G$ can be mapped onto the circle 
$|t|<1$; if $t=F(w), F(0)=0$ is a univalent function which effects such a map, then the function $t=F(f(z))$ is analytic of class (A) in $|z|<1$ with $m$ zeros. Since zero is not an asymptotic value of $F(f(z))$, it follows from Theorem 1 that $F(f(z))$ gives an $(m, 1)$ conformal mapping of $|z|<1$ onto $|t|<1$, so that $f(z)$ in (4) gives an $(m, 1)$ conformal mapping of $|z|<1$ onto the region $G$ which consists of the $w$-plane slit along an arc of $|w|=1$.

We remark that this theorem is similar to a theorem of Lehto [7, p. 12]; the example given below will show, however, that the class (B) is not contained in the class (L).

3. Functions of class (L). We assume that values which $w=f(z)$ assumes in $|z|<1$ lie in some domain $G$ of the $w$-plane whose boundary $\Gamma$ has positive capacity and $f^{*}\left(e^{i \theta}\right)$ belongs to $\Gamma$ for almost all $e^{i \theta}$ on $|z|=1$.

TheOREM 3. Let $w=f(z)$ be of class (L) in $|z|<1$ with respect to a domain $G$ in the w-plane whose boundary $\Gamma$ has positive capacity. Let $f^{*}\left(e^{i \theta}\right)$ belong to $\Gamma$ for almost all $e^{i \theta}$ on $|z|=1$. Then every arcwise accessible point of $\Gamma$ is a radial limit value of $f(z)$.

Let $D$ be that subdomain of $G$ which consists of the values which $f(z)$ assumes in $|z|<1$. It has been shown by Lehto [8, p. 97] that the set $S$ of points of $G$ not assumed by $f(z)$ is of capacity zero; obviously $G=D \cup S$.

From the fact that a set $S$ of capacity zero cannot separate the plane, it is a simple consequence that if a point $\alpha \in \Gamma=\operatorname{Fr}(G)$ is arcwise accessible from $G$ it is arcwise accessible from $D$. Indeed, let $L$ be a Jordan arc lying in $G$ and terminating at $\alpha$. Let $\kappa_{n}, n=1,2, \cdots$, be a set of circular neighborhoods lying in $G$ with centers $C_{n}$ on $L$ and radii $r_{n}$ such that as $n \rightarrow \infty, C_{n} \rightarrow \alpha$ and $r_{n} \rightarrow 0$, and such that $\kappa_{n} \cap \kappa_{n+1}$ is not empty for any $n$. Since $\kappa=U_{n=1}^{\infty} \kappa_{n}$ is open and covers $L$, there exists also another Jordan arc $J$ disjoint from $L$, lying in $\kappa$ and terminating at $\alpha$. Next pick two sequences of points, $l_{n}$ belonging to $L \cap D$ and $j_{n}$ belonging to $J \cap D$, such that as $n \rightarrow \infty, l_{n} \rightarrow \alpha$ and $j_{n} \rightarrow \alpha$. This is clearly possible since $S$ is of capacity zero and thus can contain no arc. Because $S$ cannot separate the plane, we are able to join $l_{n}$ to $j_{n}$ by an arc $\gamma_{n}$ lying in $D$ and to pick a point $w_{n}$ on $\gamma_{n}$, $l_{n} \neq w_{n} \neq j_{n}$. We join $w_{n}$ to $w_{n+1}$ by an arc $K_{n}: w=w(t), 1 /(n+1)<t$ $\leqq 1 / n$ such that $K_{n}$ lies in $D$. Since $K=\bigcup_{n=1}^{\infty} K_{n}$ is an arc: $w=w(t)$, $0<t \leqq 1$, lying in $D$ and terminating at $\alpha$, we have that $\alpha$ is arcwise accessible from $D$. Let $L_{\alpha}$ be an arc in $D$ which terminates at the point $\alpha$. Let $w=w(\zeta), w(0)=f(0)$ be a function which maps the circle 
$|\zeta|<1$ conformally onto the universal covering surface $\Omega_{D}$ of $D$; since $\Gamma$ has positive capacity, such a mapping will exist and will be of bounded characteristic in $|\zeta|<1$. If we denote by $\zeta=\zeta(w)$ that branch of the inverse function of $w=w(\zeta)$ which vanishes at $w=f(0)$, the function $\zeta=F(z)=\zeta(f(z))$ can be continued along any path lying in $|z|<1$, so that, by the monodromy theorem, $F(z)$ will be a singlevalued analytic function of class (A) in $|z|<1$.

There exists at least one point $e^{i \beta}$ on $|\zeta|=1$ and an $\operatorname{arc} L_{\beta}$ of $|\zeta|<1$ terminating at $e^{i \beta}$ such that the image of $L_{\beta}$ under $w=w(\zeta)$ is $L_{\alpha}$. Since $w=w(\zeta)$ omits at least three values, it follows from an extension of a theorem of Lindelöf $\left[2\right.$, p. 96] that the $\lim _{\zeta \rightarrow e^{i \beta} w(\zeta)=\alpha}$ exists uniformly in the angle $V_{\delta}:\left|\arg \left(1-\zeta e^{-i \beta}\right)\right| \leqq(\pi / 2)-\delta$, for any $\delta>0$. Now $\zeta=F(z)$ as a function of class (A) assumes in $|z|<1$ all values in $|\zeta|<1$ except for a set $S^{\prime}$ of capacity zero [3, p. 111]. Furthermore $F^{\prime}(z)$ is an analytic function in $|z|<1$ and, as such, possesses at most a countable number of zeros $\left\{p_{n}\right\}$. If we denote $\bigcup_{n} F\left(p_{n}\right)=Q$ we see that it is possible to find a second arc $L_{\beta}^{\prime}$ terminating at $e^{i \beta}$ and lying inside $V_{\delta}$ such that $L_{\beta}^{\prime}$ does not pass through any points of the set $S^{\prime} \cup Q$. It now follows from a well-known method (see, for example, $[1$, p. 230]) that a branch of the inverse function of $F(z)$ may be continued along $L_{\beta}^{\prime}$, so that there exists an $\operatorname{arc} L_{z}$ lying in $|z|<1$ and terminating at a point $e^{i \theta_{0}}$ of $|z|=1$ such that, as $z \rightarrow e^{i \theta_{0}}$ along $L_{z}, \zeta \rightarrow e^{i \beta}$ along $L_{\beta}^{\prime}$. From the identity $w(\zeta)=w(F(z))$ $=f(z)$, it follows that $f(z) \rightarrow \alpha$ as $z \rightarrow e^{i \theta_{0}}$ along $L_{z}$, so that by the extension of Lindelöf's theorem mentioned above $\lim _{r \rightarrow 1} f\left(r e^{i \theta_{0}}\right)=\alpha$.

We remark that every arcwise accessible point $\alpha$ of $\Gamma$ is a radial limit of $f(z)$ infinitely of ten except for two cases: (1) whenever $G$ is simply-connected and $f(z)$ effects an $(m, 1)$ conformal mapping of $|z|<1$ onto $G$; in this case, each arcwise accessible point $\alpha \in \Gamma$ is a radial limit exactly $m$ times; (2) whenever $G$ is doubly-connected and one boundary component reduces to a point, and $f(z)$ maps $|z|<1$ conformally onto the covering surface of $G$; in this case the degenerate boundary component will be a radial limit finitely often, while all other arcwise accessible boundary points of $G$ will be radial limits of $f(z)$ infinitely often. As an example of case (2) we exhibit the function $w=\exp \left(\left(z^{n}+1\right) /\left(z^{n}-1\right)\right)$ which maps $|z|<1$ in an $(n, 1)$ conformal way onto the covering surface of the disc $|w|<1$ punctured at $w=0$. Here the boundary $\Gamma$ of $G$ consists of the circumference $|w|=1$ and the point $w=0$; the degenerate boundary component, $w=0$, is a radial limit only at the $n$th roots of unity, while all points of $|w|=1$ are radial limits infinitely of ten.

We conclude this section by exhibiting a function $f(z)$ which is of 
class (B) but not of class (L); $f(z)$ is the quotient of two Blaschke products $B_{1}(z) / B_{2}(z)$, where $B_{1}(z)$ has an infinite number of zeros $\left\{z_{n}\right\}$ on the oricycle $r=\cos \theta,(0<\theta \leqq \pi / 2)$, and where $B_{2}(z)$ has its zeros at the conjugate points $\left\{\bar{z}_{n}\right\}$. We shall show that $f(z) \rightarrow 0$ as $z \rightarrow 1$ along the curve $r=\cos \theta(0<\theta \leqq \pi / 2)$ and that $f(z) \rightarrow \infty$ as $z \rightarrow 1$ along $r=\cos \theta(-\pi / 2 \leqq \theta<0)$. It will then follow from a theorem of Lindelöf $[11$, p. 67] that $f(z)$ must assume all complex values-with two possible exceptions-infinitely of ten in the region $|z-1 / 2|<1 / 2$, so that the values assumed by $f(z)$ in $|z|<1$ cannot lie in a domain whose boundary has positive capacity.

We shall simplify the construction of the Blaschke products by considering their analogues in the half-plane $R(z)>0$; by conformal mapping, the properties described in the last paragraph will then hold in the unit circle. We consider the function

$$
b_{1}(z)=\prod_{n=1}^{\infty} \frac{z-z_{n}}{z+\bar{z}_{n}}
$$

where $R\left(z_{n}\right)>0$; if $\sum_{n=1}^{\infty} R\left(1 / z_{n}\right)<\infty$ then $b_{1}(z)$ is the analogue of the Blaschke product in the right half-plane (cf. [13, p. 142]). We choose the zeros of $b_{1}(z)$ to be the numbers $z_{n}=1+i n^{t}$, where $1 / 2<t$ $<1$; clearly $\sum_{n=1}^{\infty} R\left(1 / z_{n}\right)$ converges with this choice of $t$. The zeros of $b_{1}(z)$ lie on the line $R(z)=1, J(z)>0$. We form the function

$$
b_{2}(z)=\prod_{n=1}^{\infty} \frac{z-\bar{z}_{n}}{z+z_{n}},
$$

whose zeros are the complex conjugates of the zeros of $b_{1}(z)$, and consider the function

$$
f(z)=\frac{b_{1}(z)}{b_{2}(z)}=\prod_{n=1}^{\infty} \frac{z-z_{n}}{z+\bar{z}_{n}} \frac{z+z_{n}}{z-\bar{z}_{n}} .
$$

We show that $\lim _{y \rightarrow+\infty} f(1+i y)=0$ and $\lim _{y \rightarrow-\infty} f(1+i y)=\infty$. If $z=1+i y, z_{n}=1+i n^{t}$, each factor

$$
\frac{z-z_{n}}{z+\bar{z}_{n}} \frac{z+z_{n}}{z-\bar{z}_{n}}=\frac{i\left(y-n^{t}\right)}{2+i\left(y-n^{t}\right)} \cdot \frac{2+i\left(y+n^{t}\right)}{i\left(y+n^{t}\right)}
$$

is in modulus less than 1 for all $n$ and for $y>0$, and the modulus of $w=f(1+i y)$ is less than any one of its factors. Now if $n^{t} \leqq y \leqq(n+1)^{t}$, we have that

$$
\frac{y-n^{t}}{\left|2+i\left(y-n^{t}\right)\right|} \leqq \frac{y-n^{t}}{2} \leqq \frac{(n+1)^{t}-n^{t}}{2},
$$


and $(n+1)^{t}-n^{t} \rightarrow 0$ as $n \rightarrow \infty$. Since $\lim _{n \rightarrow \infty}\left(i\left(y+n^{t}\right) / 2+i\left(y+n^{t}\right)\right)=1$, it follows that the expression in (6) tends to 0 as $y \rightarrow+\infty$. Similarly, it can be shown that if $y<0$ each factor (6) of (5) has modulus greater than 1 and tends to $\infty$ as $y \rightarrow-\infty$. Hence $f(1+i y)$ has the properties described above, and the example is completed.

Added December 6, 1955: After this paper was submitted, Theorem 3 has appeared in a later paper of Lehto, Annales Academiae Scientiarum Fennicae no. 177 (1954) p. 45; the author has heard that a third proof of this theorem has also been made by M. Ohtsuka. There has also appeared a paper of K. Noshiro, Proc. Nat. Acad. Sci. U.S.A. vol. 41 (1955) pp. 398-401 containing generalizations of these results.

\section{BIBLIOGRAPHY}

1. C. Carathéodory, Funktionentheorie I, Basel, 1950.

2. E. F. Collingwood and M. L. Cartwright, Boundary theorems for a function meromorphic in the unit circle, Acta Math. vol. 87 (1952) pp. 83-146.

3. O. Frostman, Potentiel d'équilibre et capacité des ensembles, Meddelanden från Lunds Universit ets Matematiska Seminarium vol. 3 (1935) p. 111.

4. - Sur les produits de Blaschke, Kungl. Fysiografiska Sällskapets i Lund Förhandlingar vol. 12 no. 15 (1942) pp. 1-14.

5. G. Hössjer, Über die Randwerte beschränkter Funktionen, Acta Univ. Szeged. Sect. Sci. Math. vol. 5 (1930) p. 55.

6. O. Lehto, Sur la théorie des fonctions méromorphes à caractéristique bornée, C. R. Acad. Sci. Paris vol. 236 (1953) pp. 1943-1945.

7. - On meromorphic functions whose values lie in a given domain, Annales Academiae Scientiarum Fennicae vol. 160 (1953).

8. - On the distribution of values of meromorphic functions of bounded characteristic, Acta Math. vol. 91 (1954) pp. 87-112.

9. A. J. Lohwater, The boundary values of a class of meromorphic functions, Duke Math. J. vol. 19 (1952) pp. 243-252.

10. - Les valeurs asymptotiques de quelques fonctions méromorphes dans le cercle-ınité, C. R. Acad. Sci. Paris vol. 237 (1953) pp. 16-18.

11. R. Nevanlinna, Eindeutige analytische Funktionen, 2d ed., Berlin, Springer, 1953.

12. W. Seidel, On the distribution of values of bounded analytic functions, Trans. Amer. Math. Soc. vol. 36 (1934) pp. 201-226.

13. G. P6lya and G. Szegö, Aufgaben und Lehrsätze aus der Analysis I, Berlin, Springer, 1925.

14. M. Tsuji, Fundamental theorems in potential theory, J. Math. Soc. Japan vol. 4 (1952) pp. 70-95.

\section{Iowa State College and \\ UNIVERSITY OF MichigaN}

